\title{
Clinical characteristics and the identification of novel mutations of COL1A1 and COL1A2 in 61 Chinese patients with osteogenesis imperfecta
}

\author{
HAO ZHANG, HUA YUE, CHUN WANG, WEIWEI HU, JIEMEI GU, JINWEI HE, \\ WENZHEN FU, YUNQIU HU, MIAO LI and ZHENLIN ZHANG
}

\begin{abstract}
Department of Osteoporosis and Bone Diseases, Metabolic Bone Diseases and Genetic Research Unit, Shanghai Key Clinical Center for Metabolic Disease, Shanghai Jiao Tong University Affiliated Sixth People's Hospital, Shanghai 200233, P.R. China
\end{abstract}

Received December 14, 2015; Accepted September 15, 2016

DOI: $10.3892 / \mathrm{mmr} .2016 .5835$

\begin{abstract}
Osteogenesis imperfecta (OI) is an inherited connective tissue disorder characterized by brittle bone fractures. The aim of the present study was to investigate the pathogenic gene mutation spectrum and clinical manifestations of mutations in collagen type I, alpha 1 (COL1AI) and collagen type I, alpha 2 (COL1A2) genes in Chinese patients with OI. A total of 61 unrelated Chinese OI patients with COL1A1 and COL1A2 mutations were recruited. All the exons and the exon-intron boundaries of the COL1A1 and COL1A2 genes were amplified and directly sequenced and lumbar spine bone mineral density was measured by dual-energy X-ray absorptiometry. The mutations of the 61 probands included 33 missense mutations, 8 nonsense mutations, 7 splicing variants and 13 frameshift mutations in COL1A1 and COL1A2 genes. A total of 25 novel mutations were identified, including 18 in COL1A1 and 7 in COL1A2. The mutations p.Gly257Arg, p.Gly767Ser and p.Gly821Ser in COL1A1 and p.Gly337Ser in COL1A2 may be located at a mutation hotspot for human OI due to the high repetition rate in OI patients. Family history was positive for OI in 33 probands (54\%). All probands had suffered fractures and the most common fracture site was the femur. A total of 49 probands presented with blue sclerae (80.3\%), 20 probands suffered from dentinogenesis imperfecta (32.8\%) and 1 patient
\end{abstract}

Correspondence to: Professor Zhenlin Zhang, Department of Osteoporosis and Bone Diseases, Metabolic Bone Diseases and Genetic Research Unit, Shanghai Key Clinical Center for Metabolic Disease, Shanghai Jiao Tong University Affiliated Sixth People's Hospital, 600 Yishan Road, Shanghai 200233, P.R. China E-mail:zzl2002@medmail.com.cn

Abbreviations: OI, osteogenesis imperfecta; COL1A1, collagen type I, alpha 1; COL1A2, collagen type I, alpha 2; BMD, bone mineral density; DXA, dual-energy X-ray absorptiometry

Key words: osteogenesis imperfect, collagen type I, alpha 1, collagen type I, alpha 2, mutation, Chinese had hearing loss (1.6\%). These findings may improve understanding of the pathogenic gene mutation spectrum and the clinical manifestations of mutations of COL1A1 and COL1A2 genes in Chinese patients with OI.

\section{Introduction}

Osteogenesis imperfecta (OI) is an inherited connective tissue disorder characterized by brittle bone fractures and short stature. In addition, other connective tissue disorders, including blue or gray sclera, dentinogenesis imperfecta, hyperlaxity of ligaments and skin, and progressive conductive hearing loss, are common in OI patients (1). OI patients have been classified into four types by Sillence and Rimoin (2) according to clinical characteristics and patterns of inheritance. An expanded Sillence classification was published by Rauch and Glorieux in 2004 (3). Forlino et al (4) classified OI into 11 types based on the discovery of each novel gene defect. To date, a total of 19 genes have been identified as causing OI: Collagen, type I, alpha 1 (COL1A1; MIM 120150), collagen, type I, alpha 2 (COL1A2; MIM 120160), cartilage-associated protein (CRTAP; MIM 605497), leucine- and praline-enriched proteoglycan1 (LEPRE1; MIM 610339), peptidyl-prolyl isomerase B (PPIB; MIM 123841), FK506 binding protein 10 (MIM 607063), serpin family H member 1 (MIM 600943), Wnt family member 1 (MIM 164820), bone morphogenetic protein 1 (MIM 112264), interferon induced transmembrane protein 5 (IFITM5; MIM 614757), plastin 3 (MIM 300131), procollagen-lysine, 2-oxoglutarate 5-dioxygenase 2 (MIM 601865), serpin peptidase inhibitor, clade F (alpha-2 antiplasmin, pigment epithelium derived factor), member 1 (MIM 172860), Sp7 transcription factor (MIM 606633), transmembrane protein 38B (MIM 611236), cAMP responsive element binding protein 3-like 1 (HGNC ID 18856; https://oi.gene. le.ac.uk/home.php?select_db=CREB3L1), prolyl 4-hydroxylase, beta polypeptide (MIM 176790), SEC24 family member D (MIM 607186) and Secreted protein, acidic, cysteine-rich (SPARC; MIM 182120) (5-8). Although numerous genetic causes of OI have been reported, mutations in COL1A1 and COL1A2 are responsible for $\sim 90 \%$ of OI cases (9). To date, 
the COL1A1 and COLIA2 mutations in Chinese patients with OI have been reported only in certain sporadic cases (10-14). To investigate the pathogenic gene mutation spectrum and the clinical manifestations of mutations in COL1A1 and COL1A2 genes in Chinese patients with OI requires a large sample size.

Our previous study (14) identified 56 heterozygous mutations in COLIA1 and COL1A2, including 43 mutations in COL1A1 and 13 mutations in COL1A2. Of those Chinese patients, $56.9 \%$ had OI type I, $19.0 \%$ had type III and $20.7 \%$ had type IV. In addition, the study identified 2 novel compound heterozygous mutations in the LEPRE1 gene in two probands with OI. Our other previous study identified mutations in the IFITM5 gene in four Chinese families with OI type V (15). To more accurately reflect the Chinese OI clinical characteristics and pathogenic gene mutations, the present study recruited a further $61 \mathrm{OI}$ patients from 2012 to 2015. Mutations in the COL1A1 and COL1A2 genes were identified in the 61 Chinese OI patients. Although the exact incidence of OI in China remains unknown, this is a further large report of Chinese OI cases. The aim of the present study was to investigate the pathogenic gene mutation spectrum and clinical manifestations of mutations of COL1A1 and COL1A2 genes in Chinese patients with OI. These data may be useful for future clinical diagnosis and genetic counseling.

\section{Materials and methods}

Subjects. A total of 61 unrelated probands from 61 separate families were recruited from the Department of Osteoporosis and Bone Diseases, Shanghai Jiao Tong University Affiliated Sixth People's Hospital (Shanghai, China) over a 2-year period (2012-2015). The probands came from 16 provinces of China, with the majority from Eastern cities. All patients were of Han ethnicity. A total of 410 DNA samples were collected from the 61 probands, 99 family members and 250 healthy control donors. Samples from healthy controls were sequenced to determine whether mutations occurred as polymorphisms. Clinical characteristics, including scleral hue, the presence of dentinogenesis imperfecta and hearing loss, a history of fractures, height, and OI type, were recorded. The OI type was classified in all probands according to the Sillence classification (2). None of the patients belonged to a consanguineous family or had received any treatment prior to the present study. All family members were examined by a single experienced clinician familiar with OI.

Ethics statement. The present study was approved by the Ethics Committee of Shanghai Jiao Tong University Affiliated Sixth People's Hospital. Written informed consent was obtained from all adult participants prior to recruitment. In addition, written informed consent was obtained from parents on behalf of pediatric participants. This consent procedure was approved by the Ethics Committee of the Shanghai Jiao Tong University Affiliated Sixth People's Hospital.

Collagen type I mutation analysis. Genomic DNA was extracted from $2 \mathrm{ml}$ peripheral blood samples using the QuickGene DNA whole blood kit (Kurabo Industries Ltd., Osaka, Japan) and a Nucleic Acid Isolation system (QuickGene-610L; Autogen, Inc., Holliston, MA, USA).
All exons of the COL1A1 and COL1A2 genes, including the exon-intron boundaries, were amplified by polymerase chain reaction using the primers described in our previous study (14). The genomic (AF017178.2 and AF004877.1) and mRNA (Z74615.1 and Z74616.1) sequences of the COL1AI and $C O L 1 A 2$ genes were used as reference sequences. DNA mutations were numbered according to the cDNA sequence and the A of the ATG codon was designated as nucleotide +1 . Novel mutations were identified according to the Type 1 Collagen Mutation Database (https://oi.gene.le.ac.uk/variants. php?select_db=COL1A1\&action=view_all and https://oi.gene. le.ac.uk/variants.php?select_db=COL1A2\&action=view_all). In addition, control alleles from 250 healthy individuals were sequenced to determine whether novel mutations occurred as polymorphisms.

To perform a cross-species analysis of the gene sequences of the mutations that were not substitution mutations of glycine, the Uniprot database (http://www.uniprot.org/uniprot/?quer $\mathrm{y}=\mathrm{COL} 1 \mathrm{~A} 1 \&$ sort=score and http://www.uniprot.org/uniprot $/$ ?query=COL1A2\&sort=score) was used to align 10 protein sequences, and the Clustal Omega program (http://www.ebi. ac.uk/Tools $/ \mathrm{msa} / \mathrm{clustalo} /$ ) was used to view their characteristics alongside each other. The 10 COL1A1 protein sequences compared were from human, zebrafish, Japanese common newt, chicken, African clawed frog, mouse, rat, cow, dog and cat. The 10 COL1A2 protein sequences compared were human, zebrafish, green anole or horse, chicken, African clawed frog, mouse, rat, cow, dog and cat, since the protein sequence of Japanese common newt of COL1A2 was not included in the database.

Bone densitometry. The bone mineral density (BMD; $\mathrm{g} / \mathrm{cm}^{2}$ ) of the lumbar spine (L1-L4) was measured using dual-energy $\mathrm{X}$-ray absorptiometry (DXA). Vertebrae were excluded from analysis if they were affected by fractures. All subjects were assessed using Lunar Prodigy equipment (GE Healthcare Life Sciences, Chalfont, UK). The Lunar Prodigy device was calibrated daily. The coefficient of variability of the lumbar spine DXA measurements was $1.39 \%$ (16). All DXA scans were conducted by the same trained specialist. Lumber spine BMD results were converted to age- and gender-specific Z-scores as previously described (17).

Statistical analysis. Statistical analyses were performed in SPSS software version 11.0 (SPSS, Inc., Chicago, IL, USA). Group differences in enumeration data were analyzed for significance using the chi-square test. Data with normal distribution was tested for significance using independent-samples $t$-test and data with non-normal distribution using the Mann-Whitney U test. All tests were two-sided. $\mathrm{P}<0.05$ was considered to indicate a statistically significant difference.

\section{Results}

Clinical characteristics of the 61 patients. A total of 44 probands had a mutation in the COL1Al gene, and 17 had a mutation in COL1A2 (Table I). Of the patients, 35 were male and 26 female, with a median age of 11.0 years (range, 0.4-60 years). Family history was positive for OI in 33 probands (54\%), negative for OI in 27 probands (44\%) and unknown 
Table I. Clinical findings of OI in the 61 patients.

\begin{tabular}{|c|c|c|c|c|c|c|c|c|c|}
\hline Mutation & $\begin{array}{c}\text { Sex } \\
(\mathrm{M} / \mathrm{F})\end{array}$ & $\begin{array}{c}\text { Age } \\
\text { (years) }\end{array}$ & $\mathrm{Fr}>10$ & $\begin{array}{c}\text { Blue } \\
\text { sclerae }\end{array}$ & DI & $\begin{array}{c}\text { Hearing } \\
\text { loss }\end{array}$ & $\begin{array}{l}\text { Height } \\
\text { Z score }\end{array}$ & $\begin{array}{l}\text { Weight } \\
\text { Z score }\end{array}$ & $\begin{array}{l}\text { LS BMD } \\
\text { Z score }\end{array}$ \\
\hline COL1A1 $(\mathrm{n}=44)$ & $28 / 16$ & $\begin{array}{c}11.0 \\
(4.5-22.5)\end{array}$ & $\begin{array}{c}6 \\
(13.6 \%)\end{array}$ & $\begin{array}{c}37 \\
(84.1 \%)\end{array}$ & $\begin{array}{c}15 \\
(34.1 \%)\end{array}$ & $\begin{array}{c}1 \\
(2.3 \%)\end{array}$ & $\begin{array}{c}-0.8 \\
(-2.5,0.6)\end{array}$ & $\begin{array}{c}-0.5 \\
(-1.2,2.1)\end{array}$ & $-1.0 \pm 1.4$ \\
\hline COL1A2 $(\mathrm{n}=17)$ & $7 / 10$ & $\begin{array}{c}9.0 \\
(7.0-20.0)\end{array}$ & 0 & $\begin{array}{c}12 \\
(70.6 \%)\end{array}$ & $\begin{array}{c}5 \\
(29.4 \%)\end{array}$ & 0 & $\begin{array}{c}-1.2 \\
(-2.5,-0.7)\end{array}$ & $\begin{array}{c}0.2 \\
(-1.1,0.9)\end{array}$ & $-2.7 \pm 0.6$ \\
\hline
\end{tabular}

Normally distributed data are expressed as the mean \pm standard deviation. Data that are not normally distributed are expressed as the median (inter-quartile range). M, male; F, female; Fr, fracture; DI, dentinogenesis imperfecta; LS BMD, lumbar spine bone mineral density; COL1A1, collagen type I, alpha 1; COL1A2, collagen type I, alpha 2.

in 1 proband $(2 \%)$. All probands had suffered fractures, and $13.2 \%$ of probands with COL1Al mutations had suffered $>10$ fractures (Table I). A total of 49 probands presented with blue sclerae $(80.3 \%), 20$ probands suffered from dentinogenesis imperfecta (32.8\%) and 1 patient had hearing loss (1.6\%).

COL1A1 and COL1A2 mutations. A total of 44 patients had a mutation within the COL1A1 gene, and 17 had a mutation in COL1A2 (Table I). These mutations included 33 missense mutations, 8 nonsense mutations, 7 splicing variants and 13 frameshift mutations (Table II). Almost half the probands $(42.6 \% ; 26 / 61)$ had a substitution mutation of the glycine within the Gly-X-Y triplet domain of the triple helix, of which 13 were in COL1A1 and 13 were in COL1A2. Serine substitutions were the most common in the present study $(42.3 \%)$.

In total, 25 of the mutations of 26 probands ( 2 probands had the same novel mutation in COL1A2) identified were novel: 18 in COL1A1 (Table III) and 7 in COL1A2 (Table IV). Novel mutations were identified according to the Type 1 Collagen Mutation Database. In addition, these novel mutations were not present in the 250 healthy controls. Of the 25 novel mutations, 7 missense mutations, 7 frameshift mutations and 4 nonsense mutations were identified in COL1A1, and 6 missense mutations and 1 nonsense mutation were identified in COL1A2. Of these mutations only 6 were not substitution mutations of glycine. Therefore, the gene sequences of these 6 mutations were compared across species. Although the p.Ala508Thr missense mutation of COL1Al is not at a highly conserved position, other vertebrate species including cow, dog and cat all contained alanine (Fig. 1A). The proband was classified as OI type III and COL1A2, CRTAP and LEPRE1 gene mutations were excluded. The mutation is potentially functionally damaging as alanine is a nonpolar and threonine a polar amino acid. The p.Thr766Ser (Fig. 1B) and p.Thr1298Ile (Fig. 1C) missense mutations of COL1A1, and the p.Asp37Gly (Fig. 2A), p.Arg1258His (Fig. 2B) and p.Phe4Leu (Fig. 2C) missense mutations of COL1A2 are at highly conserved positions. In addition, these missense variants were searched for in the large Exome Aggregation Consortium (ExAC) database (exac.broadinstitute.org), and the frequencies of p.Ala508Thr of COL1A1, p.Arg1258His and p.Phe4Leu missense mutations of $C O L 1 A 2$ were revealed to be $2.471 \times 10^{-5}, 2.471 \times 10^{-5}$ and $8.236 \times 10^{-6}$, respectively. The remaining three missense variants were absent from
Table II. Types of COLIA1 and COL1A2 mutations in the present study.

\begin{tabular}{ccccc}
\hline Mutation & Missense & Nonsense & Splicing & Frameshift \\
\hline COL1A1 & 17 & 7 & 7 & 13 \\
COL1A2 & 16 & 1 & 0 & 0 \\
\hline
\end{tabular}

COL1A1, collagen type I, alpha 1; COL1A2, collagen type I, alpha 2.

the ExAC database. Furthermore, the combined annotation dependent depletion (CADD) pathogenicity scores of these missense variants were high. The CADD pathogenicity score of the p.Ala508Thr, p.Thr766Ser and p.Thr1298Ile mutations of COL1A1 and the p.Aso37Gly, p.Arg1258His and p.Phe4Leu mutations of COL1A2 were 13.3, 26.6, 15.8, 17.8, 23.4 and 19.8 , respectively.

The number of fractures in patients with the missense mutation close to the carboxyl-terminal end was greater compared with patients with missense mutations close to the amino terminal. In addition, 2 probands had a p.Gly257Arg (c.769G $>$ A) mutation at exon 11 of COL1A1, which is the mutation identified in 2 unrelated patients in our previous study (14); 1 proband had a p.Gly767Ser (c.2299G>A) mutation at exon 33, which is the mutation identified in 4 unrelated patients in our previous study; 1 proband had a p.Gly821Ser (c.2461G >A) mutation at exon 37 , which is the same mutation identified in 2 unrelated patients in our previous study; and 2 probands had a p.Gly337Ser (c.1009G $>$ A) mutation at exon 19 of COL1A2, which is the same mutation identified in 1 unrelated patient in our previous study.

According to the Sillence classification, no patients in the present study had OI type II. A total of 40 patients $(65.6 \%)$ had OI type I, 11 patients $(18.0 \%)$ had type III and 10 patients $(16.4 \%)$ had type IV. As in our previous study, COL1A1 mutations were more frequent than $C O L 1 A 2$ mutations in patients with OI types I and III ( $\mathrm{P}<0.05$; Fig. 3$)$. In the COL1A1 mutation group, the most common fracture site was the femur $(n=21$; $21 \%$ of all fractures), followed by tibia/fibula $(\mathrm{n}=19 ; 19 \%)$ and radius/ulna $(n=19 ; 19 \%)$. In the COL1A2 mutation group, the most common fracture site was the femur $(n=12 ; 40 \%$ of all fractures), followed by tibia/fibula $(n=5 ; 17 \%)$, radius/ulna $(n=3 ; 10 \%)$ and humerus $(n=3 ; 10 \%)$. 


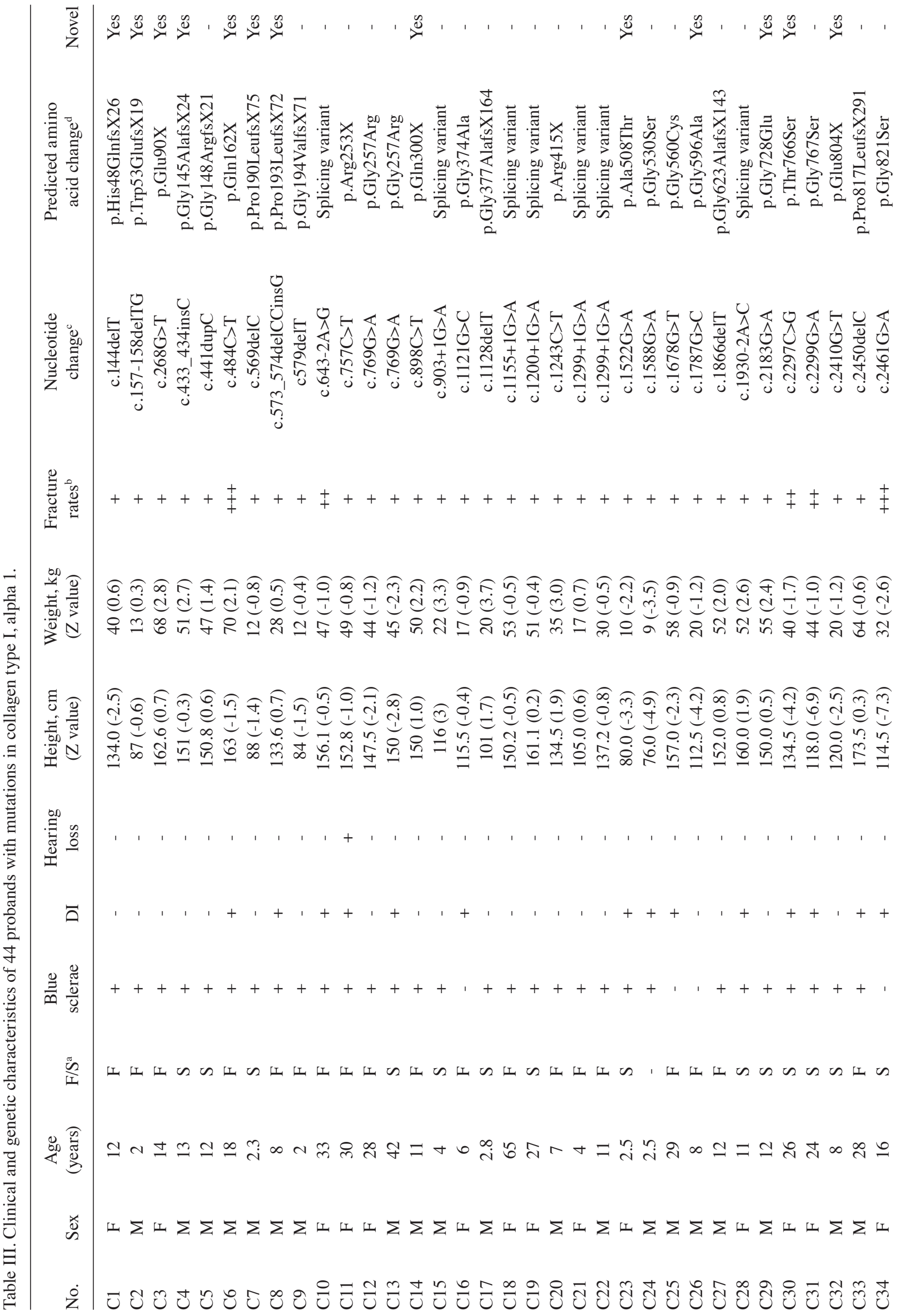




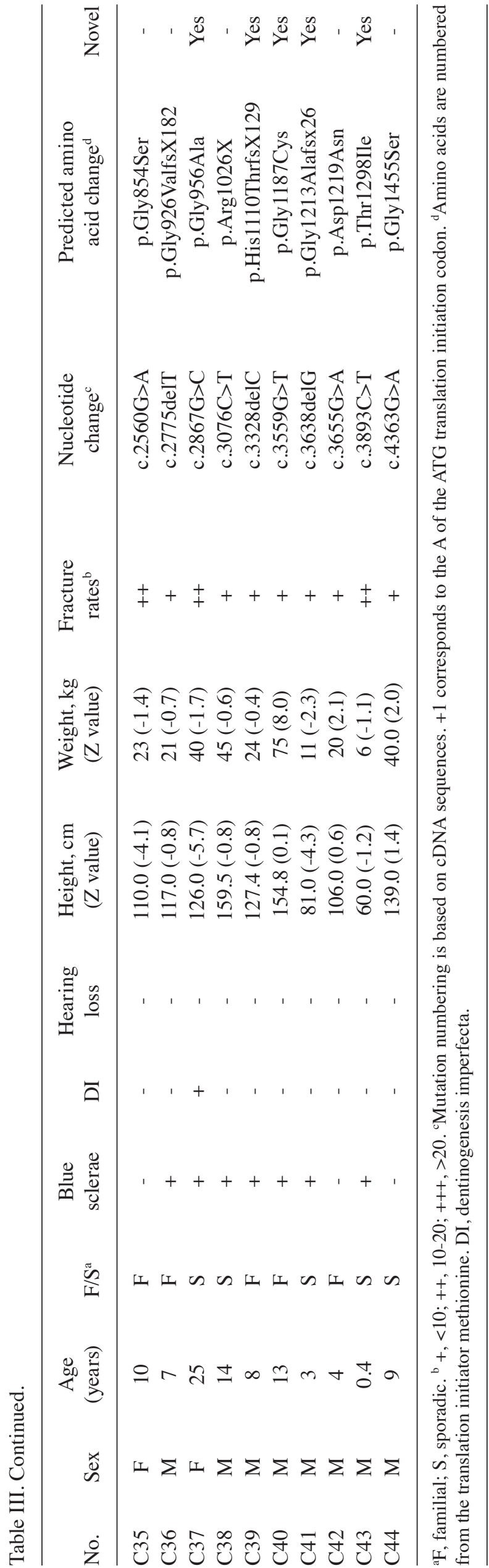

There were no significant differences in the phenotypes of patients with glycine to serine mutations of COL1A1 and COL1A2 genes (Table V).

\section{Discussion}

The present study identified 61 mutations in COL1Al and COL1A2, 25 of them novel. Of the 25 novel mutations, 13 missense mutations, 5 nonsense mutations and 7 frameshift mutations were identified. To date, 912 unique mutations in COL1A1 are listed on the OI variant database (oi.gene.le.ac. uk/home.php?select_db=COL1A1), 571 unique mutations in COL1A2 (oi.gene.le.ac.uk/home.php?select_db=COL1A2), plus a further 5 novel mutations that have recently been discovered (18-20). Despite the number of mutations identified, the 25 novel mutations observed in the present study may contribute to revealing the pathogenesis of OI and improve the disease-causing gene spectrum of OI in humans; the novel glycine substitution and frameshift mutations are of particular interest in this regard. In addition, the present study identified various misssense mutations with unclear pathogenicities, including the p.Ala508Thr, p.Thr766Ser and p.Thr1298Ile mutations of COL1A1 and the p.Asp37Gly, p.Arg1258His and p.Phe4Leu mutations of COL1A2. They occur at highly or relatively highly conserved positions and their ExAC control frequencies were very low or absent. Furthermore, the CADD pathogenicity scores of these missense variants were high. Therefore, these 6 novel misssense mutations may be pathogenic.

As in our previous study, almost three times the number of mutations was observed in COL1A1 compared with COL1A2. The COL1A1 group contains patients with stop or frameshift mutations that lead to haploinsufficiency and OI type I. However, these haploinsufficiency mutations are not observed in COL1A2, as they do not result in a phenotype.

In the present study, of the COL1A1 mutations, 4 probands had a p.Gly257Arg mutation at exon 11,5 probands had a p.Gly767Ser mutation at exon 33 and 3 probands had a p.Gly821Ser mutation at exon 37. The Type 1 Collagen Mutation Database lists these mutations 30, 26 and 21 times, respectively. In addition, 3 probands had a p.Gly337Ser mutation at exon 19 of COL1A2. The Type 1 Collagen Mutation Database lists this mutation 23 times. Therefore, these 3 mutations in COL1A1 and 1 mutation in COL1A2 may occur at a mutation hotspot for human OI.

Type I procollagen chains form a heterotrimer containing two copies of the $\alpha 1$ (I) chain and one copy of the $\alpha 2$ (I) chain; it has therefore been suggested that phenotypes resulting from mutations in COL1Al were more severe compared with those from COL1A2 (21). However, the present study observed no significant differences between COL1A1 and COL1A2 glycine to serine mutation groups, in contrast to a previous study by Rauch et al (22). The number of patients examined in the present study was small, which may limit the statistical power. As mutations toward the carboxyl-terminus are more disruptive to helix formation, it has been suggested that mutations closest to the carboxyl-terminal end would be more severe compared with those closer to the amino-terminal end of the $\alpha(\mathrm{I})$ chains $(23,24)$. However, Rauch et al (22) demonstrated this 'gradient model' of disease severity for a2(I) mutations but not for mutations affecting a1(I). In the present study, 


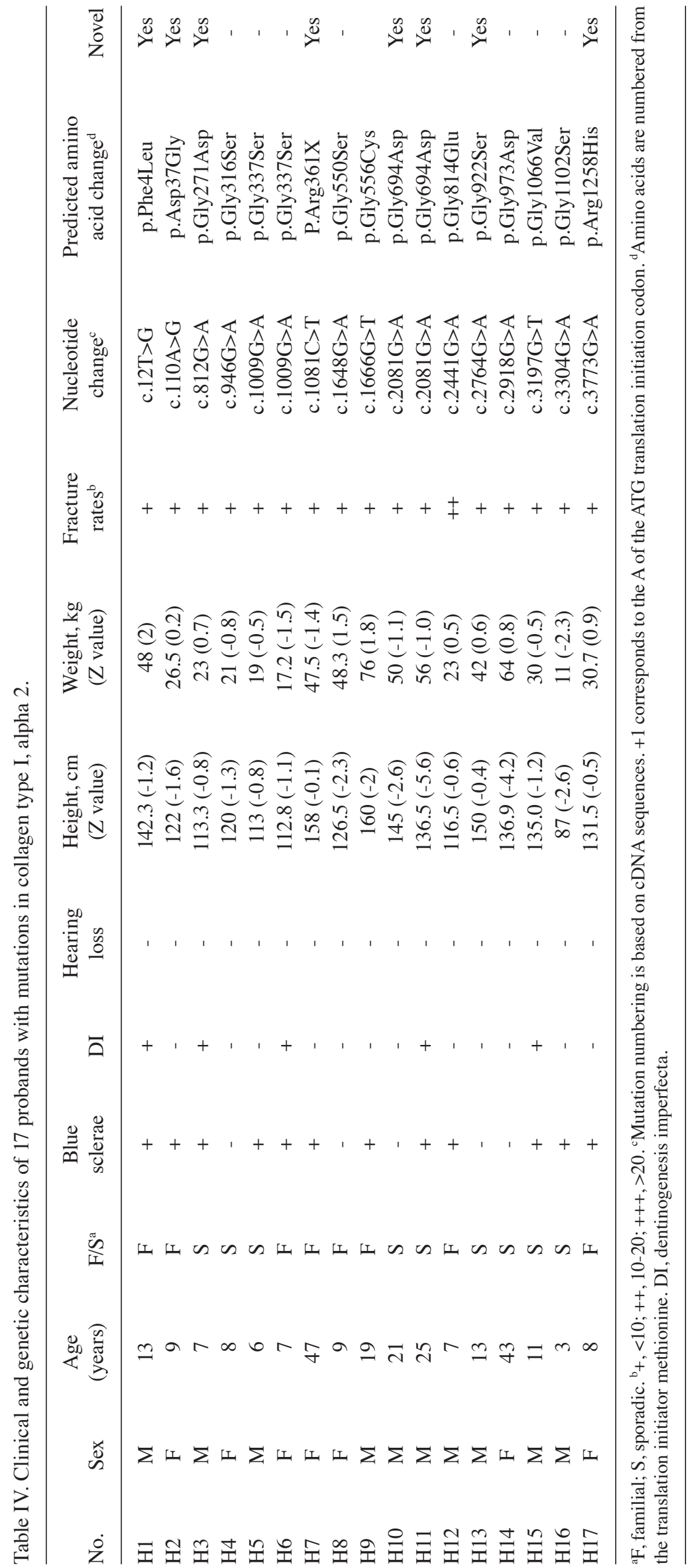




\begin{tabular}{|c|c|c|c|c|c|}
\hline A & p.Alas08Thr & B & p.Thr766Ser & $\mathrm{C}$ & p.Thr1298Ile \\
\hline Human & GVAGPKGPA GERGSPGPAGPK & Human & DGVRGITGPIGPPGPAGAPG & Human & FCNMETGETCVYPTQPSVAQK \\
\hline Zebrafish & GAAGPRGAPGERGGPGVVGPK & Zebrafish & DGIRGMI GPIGPPGPAGAPG & Zebrafish & YCNMETGET CVNPTESAIPKK \\
\hline Japanese common newt & GASGPKGAPGERGSVGPAGPK & Japanese common newt & DGARGLT GPIGPPGPSGAPG & Japanese common newt & HCNMETGETCVYPSQASISQK \\
\hline Chicken & G I AGPKGPPGERGSPGAVGPK & Chicken & DGLRGLFGPIGPPGPAGAPG & Chicken & YCNMETGETCVYPTQATIAQK \\
\hline African clawed frog & GASGPKGAPGERGPVGPAGPK & African clawed frog & DGVRGLTIFIGPPGPGGAPG & African clawed frog & YCNMETGET CIYPTQSSIPQK \\
\hline Mouse & GVAGPKGPSGERGAPGPAGPK & Mouse & DGARGITGPIGPPGPAGAPG & Mouse & YCNMETGQTCVFPTQPSVPQK \\
\hline Rat & GVAGPKGPA GERGSPGPAGPK & Rat & DGVRGITGPIGPPGPAGAPG & Rat & YCNMETGQTCVFPTQPSVPQK \\
\hline Bovine & GVAGPKGPAGERGAPGPAGPK & Bovine & DGVRGIT GPIGPPGPAGAPG & Bovine & FCNMETGE TCVYPTQPSVAQK \\
\hline Dog & GVAGPKGPA GERGSPGPAGPK & Dog & DGVRGITIGPIGPPGPAGAPG & Dog & FCNMETGEI \\
\hline Cat & GVAGPKGPAGERGSPGPAGPK & Cat & DGVRGITGPIGPPGPAGAPG & Cat & FCNMETGE TCVYPTQPHV \\
\hline
\end{tabular}

Figure 1. Missense mutations occur at conserved positions in COL1A1, as demonstrated by a comparison of the sequences of ten species. (A) The missense mutation p.Ala508Thr occurs at a relatively conserved position in COL1A1. (B) p.Thr766Ser occurs at a highly conserved position in COL1A1. (C) p.Thr1298Ile occurs at a highly conserved position in COL1A1. COL1A1, collagen type I, alpha 1.

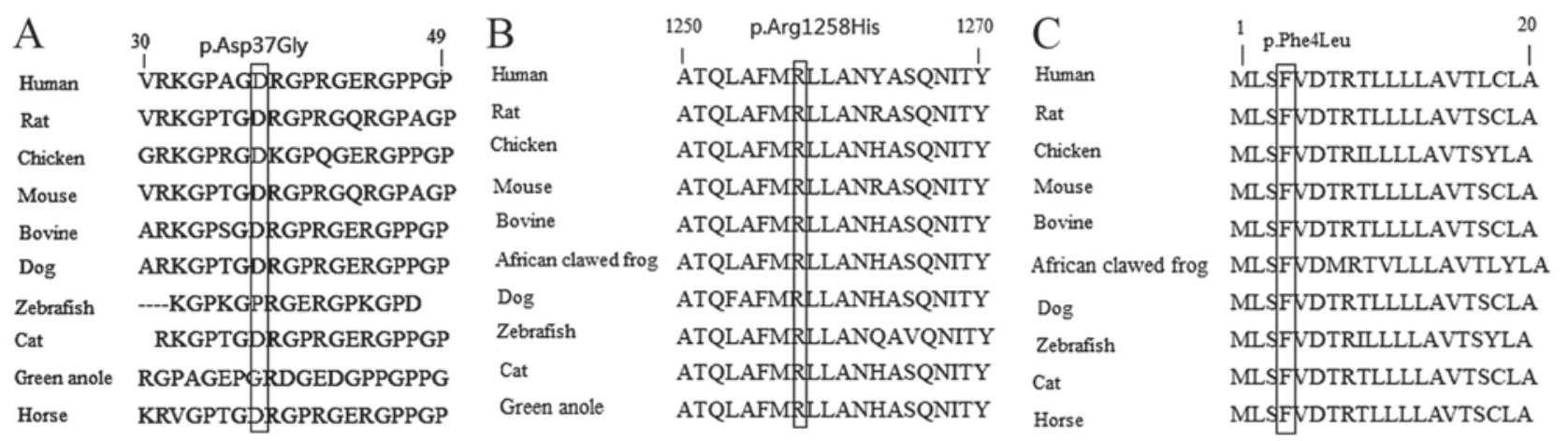

Figure 2. Missense mutations occur at conserved positions in COL1A2, as demonstrated by a comparison of the sequences of ten species. (A) The missense mutation p.Asp37Gly occurs at a highly conserved position in COL1A2. (B) p.Arg1258His occurs at a highly conserved position in COL1A2. (C) p.Phe4Leu occurs at a highly conserved position in COL1A2. COL1A2, collagen type I, alpha 2.

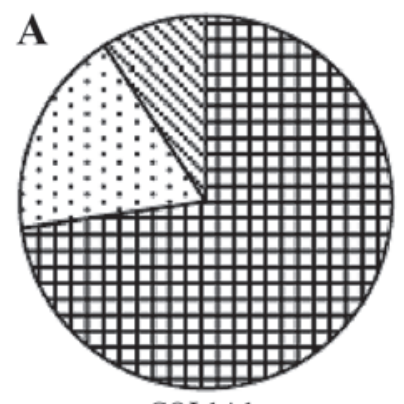

COL1A1

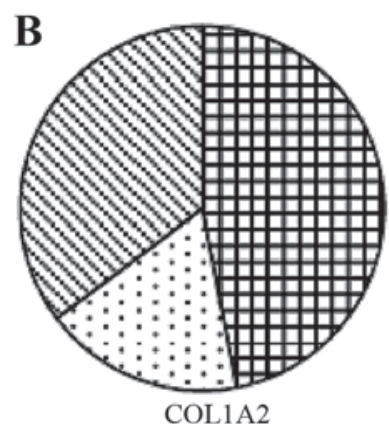

COL1A2

Figure 3. Clinical types of OI caused by COL1A1 and COL1A2 mutations (A) Clinical types of OI caused by COL1A1 mutations. (B) Clinical types of OI caused by COL1A2 mutations. Checks, OI type I; dots, OI type III stripes, OI type IV. OI, osteogenesis imperfecta; COL1A1, collagen type I, alpha 1; COL1A2, collagen type I, alpha 2.

probands with the missense mutation at the amino-terminal end had a milder phenotype compared with those closer to the carboxyl-terminal end. It has previously been demonstrated that the most common mutations associated with OI are substitutions for glycine by another amino acid in the triple helical domain of COL1A1 or COL1A2 (22). In the present study, 33 missense mutations were identified from 61 probands. Substitutions of glycine by serine were the most common in COL1A1 and COL1A2, in accordance with our previous study and Rauch et al $(14,22)$.

In contrast to our previous study, disease in the 61 patients of the present study was $54 \%$ familial and $44 \%$ sporadic disease. This was in accordance with a previous study on Korean OI patients (51\% familial and $49 \%$ sporadic OI cases) (25). In the present study, OI type I (65.6\%) was the most common type. A total of $80.3 \%$ probands presented with blue sclerae and all patients with mutations in the amino-terminal end of the a1(I) chain had blue sclera, as described by Rauch et al (22), who additionally observed that dentinogenesis imperfecta was absent in patients with mutations in the amino-terminal end of the a1(I) or a2(I) triple helical domain. In the present study, patients were identified with COLIA1 mutations at the amino-terminal and carboxyl-terminal ends without dentinogenesis imperfecta. Hearing loss is a common secondary feature of OI in adults, often with mixed conductive and sensorineural deficiency (4). Previous studies have reported that in a Scottish population $\sim 50 \%$ of patients have subjective hearing loss (26), and $>60 \%$ of Finnish OI adult patients had hearing loss (27); however, only $\sim 5 \%$ of OI pediatric patients have been reported to suffer hearing loss (28). In the present study, only $1.6 \%$ of probands had hearing loss; this may due to the fact that the majority of the patients were children. The most common fracture site was the femur, followed by tibia/fibula, radius/ulna in the patients of the COL1A1 and COL1A2 mutation groups; this is in accordance with the study by Ben Amor et al (29).

The present study is the first, to the best of our knowledge, to observe Chinese OI patients in such large numbers. 
Table V. Clinical characteristics of patients with glycine to serine substitutions.

\begin{tabular}{lccc}
\hline Parameter & $\begin{array}{c}\text { COL1A1 } \\
(\mathrm{n}=5)\end{array}$ & $\begin{array}{c}\text { COL1A2 } \\
(\mathrm{n}=6)\end{array}$ & P-value \\
\hline Age (years) & $12.3 \pm 18.1$ & $7.7 \pm 3.3$ & 0.23 \\
Height (Z score) & $-4.4 \pm 3.5$ & $-1.4 \pm 0.9$ & 0.08 \\
Weight (Z score) & $-1.3 \pm 2.1$ & $-0.5 \pm 1.4$ & 0.47 \\
\hline
\end{tabular}

Data are expressed as the mean \pm standard deviation. COL1A1, collagen type I, alpha 1; COL1A2, collagen type I, alpha 2 .

However, the present study had certain limitations. The study was limited to a single center and was cross-sectional. No patients had lethal type II OI as the patients were recruited from the outpatient center and newborns with the lethal type were not yet diagnosed.

In conclusion, the present study identified 61 mutations in COL1A1 and COL1A2, 25 of which were novel. Serine substitutions were the most frequently encountered mutation type. The mutations p.Gly257Arg, p.Gly767Ser and p.Gly821Ser in COL1A1, and p.Gly337Ser in COL1A2 may be located at a mutation hotspot for human OI. Family history was positive for OI in 33 probands (54\%). All probands had suffered fractures and the most common fracture site was the femur. A total of 49 probands presented with blue sclerae $(80.3 \%)$, 20 probands suffered from dentinogenesis imperfecta $(32.8 \%)$ and 1 patient had hearing loss $(1.6 \%)$. These findings may improve understanding of the pathogenic gene mutation spectrum and clinical manifestations of mutations in COLIAl and COL1A2 genes of Chinese patients with OI, and may be useful for future clinical diagnosis and genetic counseling.

\section{Acknowledgements}

The authors thank the patients and their family members for their invaluable cooperation, and the Center for Genetic \& Genomic Analysis, Genesky Biotechnologies Inc. (Shanghai, China) for their assistance with gene identification. The present study was supported by the National Basic Research Program of China (grant no. 2014CB942903), the Science and Technology Commission of Chongqing Municipality (grant no. CSTC2013jcyjC00009), the Science and Technology Commission of Shanghai Municipality (grant nos. 14JC1405000 and 14ZR1431900), the National Natural Science Foundation of China (grant nos. 81170803, 81370978 and 30800387), the Frontier Technology Joint Research Program of the Shanghai Municipal Hospitals (grant no. SHDC12013115) and the Shanghai Leading Talent Plan (grant no. 051).

\section{References}

1. Basel D and Steiner RD: Osteogenesis imperfecta: Recent findings shed new light on this once well-understood condition. Genet Med 11: 375-385, 2009.

2. Sillence DO and Rimoin DL: Classification of osteogenesis imperfect. Lancet 1: 1041-1042, 1978.
3. Rauch F and Glorieux FH: Osteogenesis imperfecta. Lancet 363: 1377-1385, 2004

4. Forlino A, Cabral WA, Barnes AM and Marini JC: New perspectives on osteogenesis imperfecta. Nat Rev Endocrinol 7: 540-557, 2011.

5. Van Dijk FS and Sillence DO: Osteogenesis imperfecta: Clinical diagnosis, nomenclature and severity assessment. Am J Med Genet A 164A: 1470-1481, 2014.

6. Rauch F, Fahiminiya S, Majewski J, Carrot-Zhang J, Boudko S, Glorieux F, Mort JS, Bächinger HP and Moffatt P: Cole-Carpenter syndrome is caused by a heterozygous missense mutation in P4HB. Am J Hum Genet 96: 425-431, 2015.

7. Garbes L, Kim K, Rieß A, Hoyer-Kuhn H, Beleggia F, Bevot A, Kim MJ, Huh YH, Kweon HS, Savarirayan R, et al: Mutations in SEC24D, encoding a component of the COPII machinery, cause a syndromic form of osteogenesis imperfecta. Am J Hum Genet 96: 432-439, 2015.

8. Mendoza-Londono R, Fahiminiya S, Majewski J; Care4Rare Canada Consortium; Tétreault M, Nadaf J, Kannu P, Sochett E, Howard A, Stimec J, et al: Recessive osteogenesis imperfecta caused by missense mutations in SPARC. Am J Hum Genet 96: 979-985, 2015.

9. Cheung MS and Glorieux FH: Osteogenesis imperfecta: Update on presentation and management. Rev Endocr Metab Disord 9: 153-160, 2008.

10. Liu W, Gu F, Ji J, Lu D, Li X and Ma X: A novel COL1A1 nonsense mutation causing osteogenesis imperfecta in a Chinese family. Mol Vis 13: 360-365, 2007.

11. Qin W, He JX, Shi J, Xing QH, Gao JJ, He L, Qian XQ, Liu ZJ, Shu AL and He L: Mutation detection of COL1A1 gene in a pedigree with osteogenesis imperfecta. Yi Chuan Xue Bao 32: 248-252, 2005 (In Chinese).

12. Xia XY, Cui YX, Huang YF, Pan LJ, Yang B, Wang HY, Li XJ, Shi YC, Lu HY and Zhou YC: A novel RNA-splicing mutation in COL1A1 gene causing osteogenesis imperfecta type I in a Chinese family. Clin Chim Acta 398: 148-151, 2008.

13. Lu Y, Ren X, Wang Y, Li T, Li F, Wang S, Xu C, Wu G, Li H, Li G, et al: Mutational and structural characteristics of four novel heterozygous C-propeptide mutations in the proa1(I) collagen gene in Chinese osteogenesis imperfecta patients. Clin Endocrinol (Oxf) 80: 524-531, 2014.

14. Zhang ZL, Zhang H, Ke YH, Yue H, Xiao WJ, Yu JB, Gu JM, $\mathrm{Hu}$ WW, Wang C, He JW and Fu WZ: The identification of novel mutations in COL1A1, COL1A2, and LEPRE1 genes in Chinese patients with osteogenesis imperfecta. J Bone Miner Metab 30: 69-77, 2012.

15. Zhang Z, Li M, He JW, Fu WZ, Zhang CQ and Zhang ZL: Phenotype and genotype analysis of Chinese patients with osteogenesis imperfecta type V. PLoS One 8: e72337, 2013.

16. Zhang H, He JW, Gao G, Yue H, Yu JB, Hu WW, Gu JM, Hu YQ, $\mathrm{Li}$ M, Fu WZ, et al: Polymorphisms in the HOXD4 gene are not associated with peak bone mineral density in Chinese nuclear families. Acta Pharmacol Sin 31: 977-983, 2010.

17. Maynard LM, GuoSS, Chumlea WC, Roche AF, Wisemandle WA, Zeller CM, Towne B and Siervogel RM: Total-body and regional bone mineral content and areal bone mineral density in children aged 8-18 y: The Fels Longitudinal Study. Am J Clin Nutr 68: 1111-1117, 1998.

18. Wang Y, Cui Y, Zhou X and Han J: Development of a high-throughput resequencing array for the detection of pathogenic mutations in osteogenesis imperfecta. PLoS One 10: $\mathrm{e} 0119553,2015$.

19. Hoefele J, Mayer K, Marschall C, Alberer M, Klein HG and Kirschstein M: Rare co-occurrence of osteogenesis imperfecta type I and autosomal dominant polycystic kidney disease. World J Pediatr: April 8, 2016. (Epub ahead of print). doi: 10.1007/s12519-016-0014-1.

20. Lin HY, Chuang CK, Su YN, Chen MR, Chiu HC, Niu DM and Lin SP: Genotype and phenotype analysis of Taiwanese patients with osteogenesis imperfecta. Orphanet J Rare Dis 10: 152, 2015.

21. Prockop DJ, Constantinou CD, Dombrowski KE, Hojima Y, Kadler KE, Kuivaniemi H, Tromp G and Vogel BE: Type I procollagen: The gene-protein system that harbors most of the mutations causing osteogenesis imperfecta and probably more common heritable disorders of connective tissue. Am J Med Genet 34: 60-67, 1989.

22. Rauch F, Lalic L, Roughley $P$ and Glorieux FH: Genotype-phenotype correlations in nonlethal osteogenesis imperfecta caused by mutations in the helical domain of collagen type I. Eur J Hum Genet 18: 642-647, 2010. 
23. Byers PH: Brittle bones-fragile molecules: Disorders of collagen gene structure and expression. Trends Genet 6: 293-300, 1990.

24. Marini JC, Forlino A, Cabral WA, Barnes AM, San Antonio JD, Milgrom S, Hyland JC, Körkkö J, Prockop DJ, De Paepe A, et al: Consortium for osteogenesis imperfecta mutations in the helical domain of type I collagen: Regions rich in lethal mutations align with collagen binding sites for integrins and proteoglycans. Hum Mutat 28: 209-221, 2007.

25. Lee KS, Song HR, Cho TJ, Kim HJ, Lee TM, Jin HS, Park HY, Kang S, Jung SC and Koo SK: Mutational spectrum of type I collagen genes in Korean patients with osteogenesis imperfecta. Hum Mutat 27: 599, 2006.

26. Paterson CR, Monk EA and McAllion SJ: How common is hearing impairment in osteogenesis imperfecta? J Laryngol Otol 115: 280-282, 2001
27. Kuurila K, Kaitila I, Johansson R and Grénman R: Hearing loss in Finnish adults with osteogenesis imperfecta: A nationwide survey. Ann Otol Rhinol Laryngol 111: 939-946, 2002.

28. Kuurila K, Grenman R, Johansson R and Kaitila I: Hearing loss in children with osteogenesis imperfecta. Eur J Pediatr 159: 515-519, 2000.

29. Ben Amor IM, Roughley P, Glorieux FH and Rauch F: Skeletal clinical characteristics of osteogenesis imperfecta caused by haploinsufficiency mutations in COL1A1. J Bone Miner Res 28: 2001-2007, 2013. 